\title{
The heterogeneity of the anticoagulant response to heparin
}

\section{J. WORTH ESTES}

\begin{abstract}
From the Departments of Pharmacology and Medicine, Boston University School of Medicine, Boston, Massachusetts, USA
\end{abstract}

SYNOPSIS Difficulties in evaluating the anticoagulant effect of heparin led to studies of the rate of disappearance of its effect on the whole body partial thromboplastin time, and of its simultaneously bioassayed plasma concentration. The slope of the anticoagulant's dose-effect curve varied with each subject's baseline coagulation status, which probably accounts for the wide range of clinically observed responses to the drug. The mean rate of disappearance of bioassayed heparin was less than the mean rate of disappearance of the drug's effect on the clotting time, probably because of heparin's extensive binding to the plasma proteins which comprise its site of action. That these two kinds of rate of disappearance were found not to be correlated may also reflect the proteinbinding capacity of heparin.

Although heparin is frequently the drug of choice for the emergency or prophylactic treatment of patients who have, or who may develop, thromboses, there have been difficulties in evaluating the anticoagulant's effect in the clinical laboratory. One frequent source of difficulty has been the wide range of 'normal', or baseline, clotting times, found by any clotting test, for persons not receiving anticoagulants.

In earlier studies of the problem of devising reliable and practical dosage schedules for heparin (Estes, Pelikan, and Krüger-Thiemer, 1969; Estes, 1970) the half-life of heparin's effect in the body was found to be approximately one and a half hours. When compared with several kinds of coagulation test, the whole blood activated partial thromboplastin time (WBPTT) of Blakely (1968) was found to be the most efficient and convenient measure of heparin's anticoagulant activity in the clinical laboratory (Estes, 1970). This conclusion was based on statistics derived from the kinetic parameters of heparin because it is the rate of disappearance of heparin's effect that one wants to know in designing anticoagulant therapy.

Published data pertaining to the rate of disappearance of heparin from the blood, when the amount of heparin was measured by bioassays in the form of coagulation tests, led to the conclusion that in men and dogs the half-life of heparin increased with the relative dose, ie, dose per kilogram body weight of anticoagulant administered, a property shared only by some of the coumarins Received for publication 19 May 1971. and a very few other drugs (Estes et al, 1969). On the other hand, the half-life of heparin's anticoagulant effect, ie, its prolongation of the clotting time, did not increase with dose (Estes, 1970). The experiments reported here were designed to clarify this apparent contradiction.

\section{Methods}

Single intravenous injections of heparin USP (Liquaemin sodium 10, Organon, Inc.) were given to 19 healthy medical students after overnight fasting, in relative doses ranging from 57 to 147 units per kilogram, corresponding to total doses of 3,500 and 10,000 units.

A slight modification of the whole blood partial thromboplastin time (Blakeley, 1968) was the measure of anticoagulant activity in the blood: $0.5 \mathrm{ml}$ whole blood was added to a new glass test tube $(13 \times 100$ $\mathrm{mm}$ ) containing $0.1 \mathrm{ml}$ cephaloplastin, $0.1 \mathrm{ml} 4 \%$ kaolin in $0.9 \%$ sodium chloride, and $0.1 \mathrm{ml} 0.9 \%$ saline with or without added heparin. The clotting time of the mixture was determined at $37^{\circ} \mathrm{C}$ in duplicate, and the mean whole blood partial thromboplastin time used in subsequent computations. Because the members of each pair of determinations differed by no more than 5 seconds, it was not possible to evaluate further the error of the method by multiple tests on the same sample of freshly shed blood.

Appropriate data for studying the variability of whole blood partial thromboplastin times (and 
similar clotting tests) among venous blood samples taken simultaneously from different sites, or at varying intervals, from individual subjects are nowhere available. Even if they were, their contribution to the evaluation of the data presented here presumably would be limited, because the conditions of this study were similar to those of heparin's clinical use by discontinuous intravenous injection. In no subject was there any sign of local or widespread thrombophlebitis before or as a result of any venepunctures, although any intravascular clotting that might have occurred would have been expected to have influenced the whole blood partial thromboplastin time.

Aliquots of each subject's blood, taken before injection of heparin, were added to graded amounts of heparin in the system described above so as to result in final heparin concentrations of from 1 to 4 units per $\mathrm{ml}$ of undiluted plasma, after taking into account the subject's haematocrit, and the whole blood partial thromboplastin time was determined. This served as the calibration curve for each subject, with which his whole blood partial thromboplastin time at intervals after heparin injection could be converted to units per millilitre of plasma.

Thus, the same raw data obtained from each subject provided two ways of studying the kinetics of heparin: (1) the anticoagulant effect of heparin was measured by the whole blood partial thromboplastin time, in seconds, of blood samples taken at four approximately equal intervals over a period of about three hours after heparin injection. For each subject, the fourth (last) blood sample was obtained before the partial thromboplastin time had reverted to its baseline value. (2) The concentration of the anticoagulant in the blood, in units per ml plasma, was bioassayed by interpolating the whole blood partial thromboplastin time found at each interval after injection on the in-vitro calibration curves obtained before heparin was administered.

All $19 \mathrm{log}$ whole blood partial thromboplastin times, time after injection curves, and all $19 \mathrm{log}$ heparin concentration, time after injection curves, had negative slopes statistically different from zero, and values of $F$ demonstrating that they were not alinear. The half-life of heparin was computed by conventional methods of interpolation on thesecurves.

Statistical analyses of the data were accomplished by standard methods (Snedecor, 1956). All regression lines were calculated by the method of least squares. For all procedures, statistical significance was inferred if values for $P$ were less than 0.05 .

\section{Results}

The 19 plots of log whole blood partial thrombo- plastin time and in vitro heparin concentration had positive slopes statistically different from zero, and values of $F$ demonstrating that they were not alinear. However, the slopes of the calibration curves decreased with increasing intercepts $(r=-0.667$, $P<0.01)$. That is, as Figure 1 shows, as the baseline whole blood partial thromboplastin time, with no heparin added, increased through the expected range of 85 to 141 seconds (mean \pm standard deviation $=105 \pm 13.7$ seconds), the rate of increase of the partial thromboplastin time with heparin concentration decreased. This is an instance of the influence of initial values which casts considerable doubt on the clinical applicability of standard measures of central tendency such as the mean and standard deviation for expressing 'normal' values for this clotting test, or, possibly, for others.

The half-life of heparin in the 19 subjects, determined by the bioassay, did not vary with either the slope $(r=+0.291, P<0.05)$ or the intercept $(r=-0.339, P>0.05)$ of their respective calibration curves; neither did the half-life of heparin's anticoagulant effect, determined by the whole blood partial thromboplastin time, vary with the slope $(r=+0 \cdot 175, P>0.05)$ or the intercept $(r=-0.209$, $P>0.05$ ) of the calibration curves for the bioassays.

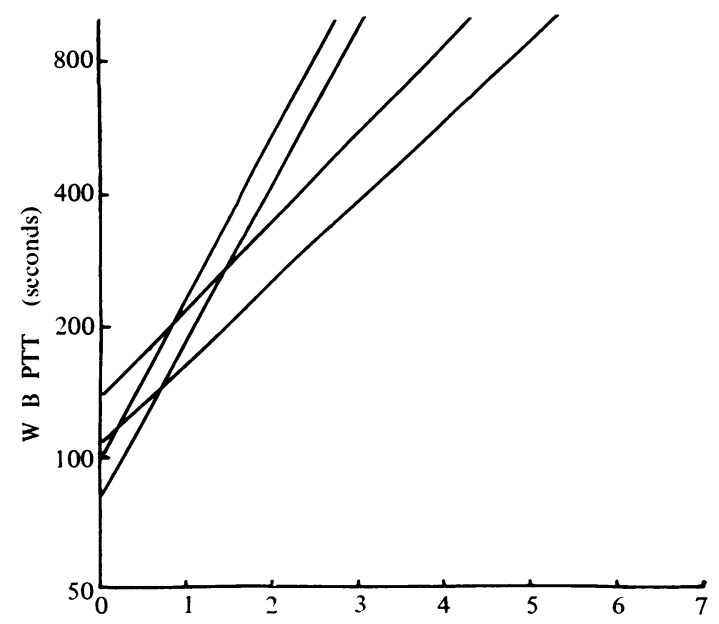

Heparin concentration in vitro $(\mathrm{u} / \mathrm{ml}$ plasma $)$

Fig. 1 Regression lines showing the relationship between the whole blood activated partial thromboplastin time (WBPTT) and heparin concentration in vitro, used to bioassay plasma concentrations of heparin in vivo. The four lines shown are the curves with the smallest and largest $Y$ intercepts, and those which intersect the smallest and largest values of $X$ on an imaginary line drawn parallel to the abscissa at 800 seconds. Thus, the other 15 calibration curves obtained in the study fall within these limits. 
Therefore, although the subject's clotting mechanisms were affected by heparin in vitro to different degrees, depending on their baseline partial thromboplastin times, the properties of his own dose-effect curve did not affect the kinetics of bioassayed heparin, or of its anticoagulant effect, for each subject.

The in vitro dose-effect calibration curves suggest that, because of the differences among the properties of the subjects' coagulation mechanisms, a given value of the whole blood partial thromboplastin time for one heparinized subject may not reflect a change in that subject's coagulation status that is necessarily or predictably of the same magnitude as that for another subject with the same value (see Figure 1). This lack of predictive value demonstrates another problem in evaluating a single clotting time in a heparinized patient, even when his pre-heparin, baseline, whole blood partial thromboplastin time is known.

Figure 2 shows that when the half-life of heparin was measured by the bioassay, the half-life increased with relative dose, as previously published bioassay data had shown (Estes et al, 1969). Figure 2 also shows that the half-life of heparin's anticoagulant effect did not increase with dose in the same subjects, as we had found previously using four different measures of heparin effect (Estes, 1970). Furthermore, the half-lives of heparin calculated from the two ways of using the same raw data from the same 19 subjects were found not to be correlated with each other $(r=+0.421, P>0.05)$, even though the bioassay data were derived from the data pertaining to the disappearance of heparin's anticoagulant effect. Therefore, it cannot be assumed that the rate of disappearance of the effect of heparin on the whole blood partial thromboplastin time predicts the rate of its disappearance from the blood when bioassayed by the whole blood partial thromboplastin time.

The mean half-life of heparin measured by bioassay $(1.387 \pm$ standard error $0.143 \mathrm{hr})$ is about $80 \%$ of the mean half-life of heparin's anticoagulant effect $(1.735 \pm \mathrm{SE} 0.100 \mathrm{hr})$; the $\mathrm{t}$ test shows this difference to be statistically significant $(P<0.025)$. Similarly, heparin's mean half-life computed from the retrospective bioassay data $(1.458 \pm$ SE 0.012 hr) (Estes et al, 1969) is about $85 \%$ of the mean half-life of heparin's anticoagulant effect (Estes, 1970 ), although the $15 \%$ difference is not statistically remarkable.

\section{Discussion}

It is not yet possible to ascertain whether the halflife of heparin determined by the bioassay method
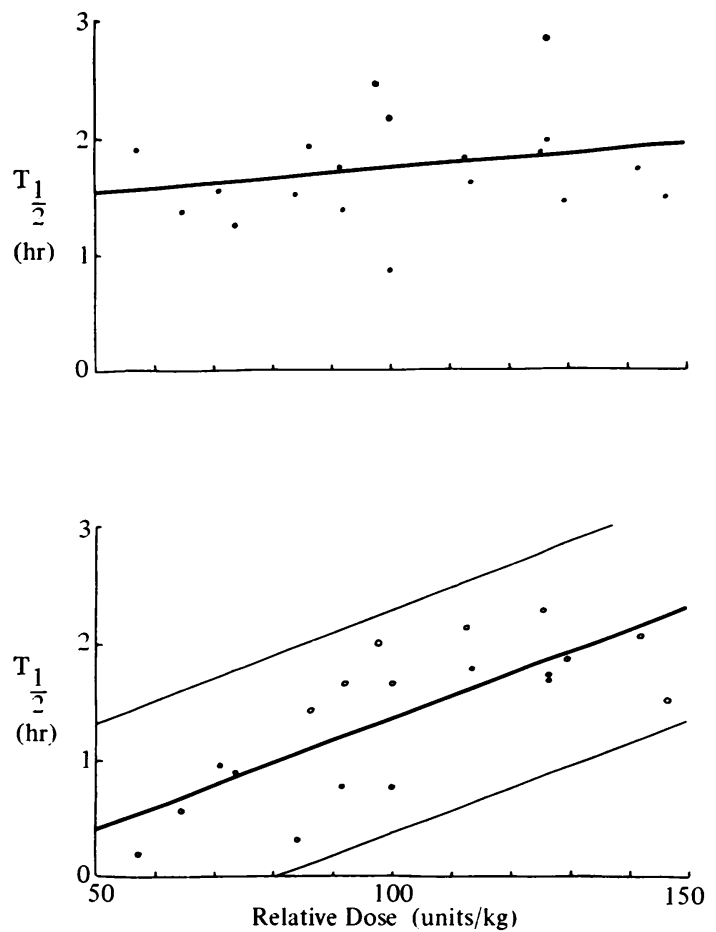

Fig. 2 Regression lines showing the relationship between the dose of heparin administered and its holf-life measured by its anticoagulant effect on the whole blood activated partial thromboplastin time (WBPTT) $(r=+0.207, \mathrm{P}<0.05$, upper curve $)$, and by bioassay $(r=+0.758, \mathrm{P}<0.01$, lower curve) .

or its half-life determined by the rate of disappearance of heparin's anticoagulant effect is the 'correct' half-life of the drug. It follows that the apparent dependence of the half-life of bioassayed heparin on its dose cannot be proven until a reliable assay method that does not take into account its biological activity is available.

The addition of heparin to blood in vitro may cause secondary changes in plasma proteins, including heparin's substrates, that are not equivalent to any changes that might result from adding heparin to blood in vivo. This possibility is suggested by the combination of the heterogeneous responses in vitro to heparin, ie, the dependence of the slope of the dose-effect curve on each subject's baseline whole blood partial thromboplastin time, with the lack of correlation between the half-lives determined by bioassay and by disappearance of anticoagulant effect.

The reactions of heparin with proteins of several kinds, and the dissociation constants of those reactions, have not yet been elucidated (Jaques, 
1943; Braswell, 1968) because of the diverse sizes of the molecular fragments that constitute this strongly electro-negative mucopolysaccharide. Chemical analyses of the samples used for bioassays in this study, with both a dye-binding method (Bassiouni, 1953) and by degradation to uronic acid (Bitter and Muir, 1962), have not been adequately reproducible, probably because heparin is strongly bound to proteins (Hoch and Chanutin, 1952).

Abildgaard $(1968 \mathrm{a}, \mathrm{b})$ has suggested that heparin exerts its anticoagulant effect by reversibly binding fibrinogen and thrombin, thereby inhibiting thrombin's usual catalytic role in the formation of fibrin. The same thrombin-binding property has been thought to account for heparin's ability to inhibit the activation of factor IX by factor XI (Shanberge and Fukui, 1967). Heparin also synergizes, or catalyzes, the action of a naturally occurring alpha-2 globulin called heparin plasma cofactor, which by itself exerts a slowly progressive heparin-like effect, in contrast to the immediate effect of heparin (Abildgaard, 1968a, b).

That the half-life of bioassayed heparin is less than that of heparin's anticoagulant effect is apparent when both the previously published bioassay data (Estes et al, 1969) and the bioassay data presented here are compared with the rates of return toward baseline clotting times presented here and earlier (Estes, 1970). It would be anticipated that the drug's effect would disappear more slowly than the drug itself if it is bound to its site of action in the plasma. Heparin is, in fact, known to be extensively bound to globulins and to fibrinogen (Hoch and Chanutin, 1952). Its spectrum of protein binding would also account for the distribution of heparin in the plasma volume that was inferred in the retrospectively analysed data, although under certain circumstances heparin can cross endothelial membranes (Yoshimura and Djerassi, 1962) and gain access to the extravascular compartment.

The usual clinical practice has been to adjust the dosage of heparin to the whole blood clotting time. The data reported here suggest, however, that although the whole blood clotting time or, alternatively, the partial thromboplastin time, provide an estimate of the plasma concentration of the drug, the relationship varies from subject to subject. Because the duration of drug effect varies with the concentration of heparin at a given time, and with its half-life-a value which cannot be determined from a single estimate of plasma heparin concentration, however precise it might be- the duration of action of a dose of heparin cannot be predicted from a single clotting time (or whole blood partial thromboplastin time) determination. Adjustment of dosage according to the results of a single estimation is unlikely to permit successful achievement of a given duration and intensity of heparin effect, because a single determination cannot predict the concentration of anticoagulant at any future time: at least two determinations are required to define the rate of disappearance of any drug.

The dependence of the properties of heparin's dose-effect curve in vitro on the subject's pre-heparin clotting status suggests that setting an arbitrary degree of prolongation of the clotting time of the whole blood partial thromboplastin time as the criterion for heparin effect will not result in reproducible and predictable degrees of anticoagulation among patients receiving the drug. That is, although prolongation of the clotting time by two or two and a half times may well indicate that the patient is anticoagulated, the same clotting time may reflect different degrees of anticoagulation in different patients.

Using these data, we are now studying the reliability and practicability of controlling heparin therapy by determining each patient's anticoagulant calibration curve with the whole blood partial thromboplastin time before beginning heparin therapy, followed by at least two determinations (which are statistically sufficient) following each of several successive heparin injections.

This work was supported by grant-in-aid no. 903 from the Northeast Chapter of the Massachusetts Heart Association.

Dr George Stidworthy of the Bedford, Massachusetts, Veterans Administration Hospital, kindly performed the uronic acid assays.

\section{References}

Abildgaard, U. (1968a). Inhibition of the thrombin-fibrinogen reaction by heparin in the absence of cofactor. Scand. J. Haemat., 5 , 432-439.

Abildgaard, U. (1968b). Inhibition of the thrombin-fibrinogen reaction by heparin and purified cofactor. Scand. J. Haemat., 5, 440-453.

Bassiouni, M. (1953). The estimation of heparin in blood. J. clin. Path., 6, 39-40.

Bitter, T., and Muir, H. M. (1962). A modified uronic acid carbazole reaction. Analyt. Biochem., 4, 330-334.

Blakely, J. A. (1968). A rapid bedside method for the control of heparin therapy. Canad. med. Ass.J., 99, 1072-1076.

Braswell, E. (1968). Heparin: molecular weight and degradation studies. Biochem. biophys. Acta (Amst.), 158, 103-116.

Estes, J. W. (1970). Kinetics of the anticoagulant effect of heparin. J. Amer. med. Ass., 212, 1492-1495.

Estes, J. W., Pelikan, E. W., and Krüger-Thiemer, E. (1969). A retrospective study of the pharmacokinetics of heparin. Clin. Pharmacol. Ther., 10, 329-337.

Hoch, H., and Chanutin, A. (1952). Effects of anticoagulants on serum and plasma. J biol Chem , 197, 503-511.

Jaques, L. B. (1943). The reaction of heparin with proteins and complex bases. Biochem. J., 37, 189-195.

Shanberge, J. N., and Fukui, H. (1967). Studies on the anticoagulant action of heparin, protamine, and Polybrene in the activation of factor IX. J. Lab. clin. Med., 69, 927-937.

Snedecor, G. W. (1956). Statistical Methods, 5th ed., The Iowa State University Press, Ames, Iowa.

Yoshimura, H., and Djerassi, I. (1962). Blood coagulation and vas cular integrity: effects of heparin. Blood, 20, 602-608. 